\title{
Interpretation of particle number size distributions measured across an urban area during the FASTER campaign
}

\section{Roy M. Harrison et al.}

Correspondence to: Roy M. Harrison (r.m.harrison@bham.ac.uk)

The copyright of individual parts of the supplement might differ from the CC BY 4.0 License. 
11 Table S1: Lognormal modes fitted to average size distribution (eqn 1) for each wind direction sector $\left(^{\circ}\right.$, measured at LHR, value is midpoint of $45^{\circ}$

12 sector). Location refers to the diameter, and FWHM is Full Width at Half Maximum (nm).

\section{BT Tower}

\begin{tabular}{|c|c|c|c|c|c|}
\hline \multicolumn{6}{|l|}{ First mode } \\
\hline $\begin{array}{c}\text { Wind } \\
\text { Direction }\end{array}$ & $\begin{array}{l}\text { Number of } \\
\text { observations } \\
\text { per wind } \\
\text { sector }\end{array}$ & $\begin{array}{l}\text { Location } \\
(\mathrm{nm})\end{array}$ & Amplitude & $\begin{array}{c}\text { Area } \\
\mathbf{n m} \\
\mathbf{c m}^{-3}\end{array}$ & FWHM \\
\hline 0 & 46 & 25.35 & 3928.49 & 234104 & 48.51 \\
\hline 45 & 86 & 28.66 & 4067.12 & 361898 & 68.13 \\
\hline 90 & 90 & 25.22 & 3915.02 & 229514 & 47.83 \\
\hline 135 & 83 & 24.38 & 5330.23 & 202014 & 32.81 \\
\hline 180 & 91 & 23.83 & 5580.83 & 237266 & 36.25 \\
\hline 225 & 42 & 40.33 & 6276.17 & 759237 & 93.61 \\
\hline 270 & 22 & 24.04 & 8859.68 & 583838 & 51.98 \\
\hline 315 & 28 & 32.42 & 4776.51 & 455667 & 74.1 \\
\hline
\end{tabular}

\begin{tabular}{|c|c|c|c|c|c|}
\hline \multicolumn{6}{|l|}{$\begin{array}{l}\text { Second } \\
\text { mode }\end{array}$} \\
\hline $\begin{array}{c}\text { Wind } \\
\text { Direction }\end{array}$ & $\begin{array}{c}\text { Number of } \\
\text { observations } \\
\text { per wind } \\
\text { sector }\end{array}$ & $\begin{array}{c}\text { Location } \\
(\mathrm{nm})\end{array}$ & Amplitude & Area & FWHM \\
\hline 0 & 46 & 90.17 & 3169.93 & 522734 & 140.16 \\
\hline 45 & 86 & 115.67 & 2050.77 & 382737 & 161.42 \\
\hline 90 & 90 & 89.7 & 3202.53 & 527251 & 139.85 \\
\hline 135 & 83 & 69.49 & 6570.34 & 825761 & 110.16 \\
\hline 180 & 91 & 66.22 & 3539.76 & 310627 & 77.59 \\
\hline 225 & 42 & & & & \\
\hline 270 & 22 & 107.06 & 2413 & 464582 & 164.11 \\
\hline 315 & 28 & 118.145 & 1854.66 & 392172 & 180.56 \\
\hline
\end{tabular}




\begin{tabular}{|c|c|c|c|c|c|}
\hline \multicolumn{6}{|l|}{ Third mode } \\
\hline $\begin{array}{l}\text { Wind } \\
\text { Direction }\end{array}$ & $\begin{array}{l}\text { Number of } \\
\text { observations } \\
\text { per wind } \\
\text { sector }\end{array}$ & $\begin{array}{l}\text { Location } \\
\text { (nm) }\end{array}$ & Amplitude & Area & FWHM \\
\hline 0 & 46 & 252.39 & 1083.55 & 256315 & 214.96 \\
\hline 45 & 86 & 255.28 & 1062.52 & 256633 & 219.37 \\
\hline 90 & 90 & 252.27 & 1083.66 & 256300 & 214.92 \\
\hline 135 & 83 & 192.92 & 999.77 & 231929 & 207.04 \\
\hline 180 & 91 & 163.22 & 1615.88 & 218842 & 176 \\
\hline 225 & 42 & & & & \\
\hline 270 & 22 & & & & \\
\hline 315 & 28 & & & & \\
\hline
\end{tabular}




\begin{tabular}{|c|c|c|c|c|c|}
\hline \multicolumn{6}{|l|}{ First mode } \\
\hline $\begin{array}{c}\text { Wind } \\
\text { Direction }\end{array}$ & $\begin{array}{c}\text { Number of } \\
\text { observations } \\
\text { per wind } \\
\text { sector }\end{array}$ & $\begin{array}{l}\text { Location } \\
\quad(\mathrm{nm})\end{array}$ & Amplitude & 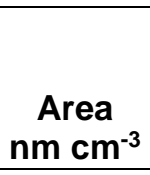 & FWHM \\
\hline 0 & 44 & 20.57 & 5126.57 & 278786 & 43.24 \\
\hline 45 & 85 & 16.97 & 4003.494 & 506720 & 27.22 \\
\hline 90 & 87 & 30.62 & 8629.39 & 841799 & 74.37 \\
\hline 135 & 80 & 21.56 & 10656.4 & 761568 & 53.67 \\
\hline 180 & 87 & 14.38 & 8390.3 & 232405 & 23.34 \\
\hline 225 & 37 & 8.59 & 4391.335 & 55150 & 10.98 \\
\hline 270 & 19 & 11.671 & 7877.21 & 119545 & 13.44 \\
\hline 315 & 30 & 22.83 & 5396.65 & 445362 & 60.92 \\
\hline
\end{tabular}

\begin{tabular}{|c|c|c|c|c|c|}
\hline $\begin{array}{c}\text { Second } \\
\text { mode }\end{array}$ & $\begin{array}{c}\text { Number of } \\
\text { Wind } \\
\text { observations } \\
\text { per wind } \\
\text { sector }\end{array}$ & $\begin{array}{c}\text { Location } \\
\text { (nm) }\end{array}$ & Amplitude & Area & FWHM \\
\hline 0 & 44 & 82.12 & 3047.83 & 415980 & 117.54 \\
\hline 45 & 85 & 70.84 & 3102.88 & 221919 & 133.59 \\
\hline 90 & 87 & 143.12 & 1417.51 & 331597 & 202.21 \\
\hline 135 & 80 & 81 & 4228.59 & 711679 & 140.22 \\
\hline 180 & 87 & 52.76 & 5815.76 & 807440 & 110.6 \\
\hline 225 & 37 & 28.59 & 7083.26 & 618987 & 67.27 \\
\hline 270 & 19 & 32.69 & 7246.06 & 716049 & 76.28 \\
\hline 315 & 30 & 81.57 & 2827.28 & 509009 & 148.51 \\
\hline
\end{tabular}




\begin{tabular}{|c|c|c|c|c|c|}
\hline \multicolumn{6}{|l|}{ Third mode } \\
\hline $\begin{array}{c}\text { Wind } \\
\text { Direction }\end{array}$ & $\begin{array}{l}\text { Number of } \\
\text { observations } \\
\text { per wind } \\
\text { sector }\end{array}$ & $\begin{array}{l}\text { Location } \\
\text { (nm) }\end{array}$ & Amplitude & Area & FWHM \\
\hline 0 & 44 & 249.69 & 1019.65 & 255664 & 226.915 \\
\hline 45 & 85 & 248.3 & 875.56 & 129033 & 229.14 \\
\hline 90 & 87 & 278.84 & 319.75 & 68855.3 & 197.61 \\
\hline 135 & 80 & & & & \\
\hline 180 & 87 & & & & \\
\hline 225 & 37 & & & & \\
\hline 270 & 19 & & & & \\
\hline 315 & 30 & & & & \\
\hline
\end{tabular}


North Kensington

\begin{tabular}{|c|c|c|c|c|c|}
\hline \multicolumn{6}{|l|}{ First mode } \\
\hline $\begin{array}{c}\text { Wind } \\
\text { Direction }\end{array}$ & $\begin{array}{l}\text { Number of } \\
\text { observations } \\
\text { per wind } \\
\text { sector }\end{array}$ & $\begin{array}{c}\text { Location } \\
(\mathrm{nm})\end{array}$ & Amplitude & 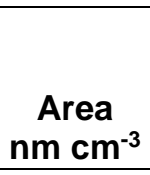 & FWHM \\
\hline 0 & 44 & 25.16 & 3677.62 & 68003 & 17 \\
\hline 45 & 85 & 25.07 & $28-4.69$ & 53038 & 17.37 \\
\hline 90 & 87 & 25.45 & 3839.42 & 69211 & 16.6 \\
\hline 135 & 79 & 23.59 & 3586.46 & 47019 & 12.16 \\
\hline 180 & 81 & 24.2 & 2504.2 & 39961 & 14.73 \\
\hline 225 & 27 & 22.37 & 2443.1 & 24056 & 9.17 \\
\hline 270 & 21 & 23.54 & 5533.2 & 98390 & 16.33 \\
\hline 315 & 28 & 26.05 & 4620.27 & 96761 & 19.18 \\
\hline
\end{tabular}

\begin{tabular}{|c|c|c|c|c|c|}
\hline $\begin{array}{c}\text { Second } \\
\text { mode }\end{array}$ & $\begin{array}{c}\text { Number of } \\
\text { Wind } \\
\text { observations } \\
\text { per wind } \\
\text { sector }\end{array}$ & $\begin{array}{c}\text { Location } \\
\text { (nm) }\end{array}$ & Amplitude & Area & FWHM \\
\hline 0 & 44 & 57 & 4922.5 & 558961 & 95.2 \\
\hline 45 & 85 & 57.62 & 3387.15 & 395938 & 97.71 \\
\hline 90 & 87 & 47.79 & 6516.87 & 450853 & 60.74 \\
\hline 135 & 79 & 36.53 & 4261.6 & 163946 & 34.68 \\
\hline 180 & 81 & 46.63 & 4150.9 & 275245 & 58.19 \\
\hline 225 & 27 & 32.96 & 4337.3 & 122760 & 25.84 \\
\hline 270 & 21 & 40.56 & 7844.84 & 451356 & 50.51 \\
\hline 315 & 28 & 52.8 & 6519.26 & 529691 & 70.66 \\
\hline
\end{tabular}




\begin{tabular}{|c|c|c|c|c|c|}
\hline Third mode & \multicolumn{5}{|c|}{} \\
\hline $\begin{array}{c}\text { Wind } \\
\text { Direction }\end{array}$ & $\begin{array}{c}\text { Number of } \\
\text { observations } \\
\text { per wind } \\
\text { sector }\end{array}$ & $\begin{array}{c}\text { Location } \\
\text { (nm) }\end{array}$ & Amplitude & Area & FWHM \\
\hline 0 & 44 & 224.9 & 970.54 & 228005 & 211.9 \\
\hline 45 & 85 & 216.54 & 1059.13 & 251998 & 213.93 \\
\hline 90 & 87 & 143.93 & 2319.51 & 508160 & 190.92 \\
\hline 135 & 79 & 73.95 & 5745.6 & 887776 & 128.58 \\
\hline 180 & 81 & 125.15 & 1756.3 & 318387 & 158.81 \\
\hline 225 & 27 & 63.1 & 5216.349 & 664931 & 106.64 \\
\hline 270 & 21 & 106.98 & 2366.82 & 425075 & 154.514 \\
\hline 315 & 28 & 131.62 & 1770.74 & 363740 & 178.437 \\
\hline
\end{tabular}




\begin{tabular}{|c|c|c|c|c|c|}
\hline \multicolumn{6}{|l|}{ First mode } \\
\hline $\begin{array}{c}\text { Wind } \\
\text { Direction }\end{array}$ & $\begin{array}{l}\text { Number of } \\
\text { observations } \\
\text { per wind } \\
\text { sector }\end{array}$ & $\begin{array}{c}\text { Location } \\
\text { (nm) }\end{array}$ & Amplitude & 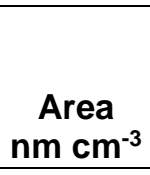 & FWHM \\
\hline 0 & 33 & 22.88 & 3691.19 & 72084.7 & 17.83 \\
\hline 45 & 75 & 22.89 & 3771.4 & 84406.1 & 20.28 \\
\hline 90 & 83 & 23.54 & 7924.86 & 152534 & 17.61 \\
\hline 135 & 76 & 21.83 & 5367.5 & 60459.1 & 10.46 \\
\hline 180 & 79 & 22.08 & 8296.72 & 112315 & 12.52 \\
\hline 225 & 34 & 20.79 & 7178.56 & 67461.8 & 8.75 \\
\hline 270 & 18 & 24.07 & 12222 & 273331 & 20.32 \\
\hline 315 & 21 & 20.94 & 2866.11 & 35984 & 11.62 \\
\hline
\end{tabular}

\begin{tabular}{|c|c|c|c|c|c|}
\hline \multicolumn{6}{|l|}{$\begin{array}{l}\text { Second } \\
\text { mode }\end{array}$} \\
\hline $\begin{array}{c}\text { Wind } \\
\text { Direction }\end{array}$ & $\begin{array}{l}\text { Number of } \\
\text { observations } \\
\text { per wind } \\
\text { sector }\end{array}$ & $\begin{array}{c}\text { Location } \\
\text { (nm) }\end{array}$ & Amplitude & Area & FWHM \\
\hline 0 & 33 & 56.62 & 4834.02 & 724755 & 119.34 \\
\hline 45 & 75 & 63.48 & 4079.18 & 634362 & 125.74 \\
\hline 90 & 83 & 48.13 & 10413.3 & 942478 & 76.57 \\
\hline 135 & 76 & 31.47 & 7858.62 & 257815 & 29.6 \\
\hline 180 & 79 & 32.24 & 9933.89 & 395955 & 35.57 \\
\hline 225 & 34 & 27.91 & 10344.56 & 258991 & 22.81 \\
\hline 270 & 18 & 54.85 & 13664.8 & 1879800 & 110.66 \\
\hline 315 & 21 & 29.46 & 4554.66 & 154631 & 30.4 \\
\hline
\end{tabular}




\begin{tabular}{|c|c|c|c|c|c|}
\hline Third mode & \multicolumn{5}{|c|}{} \\
\hline $\begin{array}{c}\text { Wind } \\
\text { Direction }\end{array}$ & $\begin{array}{c}\text { Number of } \\
\text { observations } \\
\text { per wind } \\
\text { sector }\end{array}$ & $\begin{array}{c}\text { Location } \\
\text { (nm) }\end{array}$ & Amplitude & Area & FWHM \\
\hline 0 & 33 & 263.19 & 864.15 & 198503 & 209.57 \\
\hline 45 & 75 & 257.32 & 903.69 & 222882 & 223.84 \\
\hline 90 & 83 & 159.57 & 2495.6 & 601853 & 210.5 \\
\hline 135 & 76 & 63.65 & 11942.15 & 1756280 & 120.21 \\
\hline 180 & 79 & 76.12 & 9900.46 & 1535980 & 129.64 \\
\hline 225 & 34 & 60.01 & 12575.8 & 1662990 & 108.96 \\
\hline 270 & 18 & & & & \\
\hline 315 & 21 & 66.06 & 6338.68 & 970765 & 125.12 \\
\hline
\end{tabular}

44 
Westminster University

\begin{tabular}{|c|c|c|c|c|c|}
\hline \multicolumn{7}{|c|}{$\begin{array}{c}\text { First mode } \\
\begin{array}{c}\text { Wind } \\
\text { Direction }\end{array}\end{array}$} & $\begin{array}{c}\text { Number of } \\
\text { observations } \\
\text { per wind } \\
\text { sector }\end{array}$ & $\begin{array}{c}\text { Location } \\
\text { (nm) }\end{array}$ & Amplitude & $\begin{array}{c}\text { Area } \\
\text { nm cm } \mathbf{~ c m}^{-3}\end{array}$ & FWHM \\
\hline 0 & & & & & \\
\hline 45 & 2 & 21.58 & 5152 & 52139 & 9.42 \\
\hline 90 & 27 & 23.6 & 8396.8 & 118970 & 13.1 \\
\hline 135 & 16 & 24.5 & 13092.5 & 179378 & 12.7 \\
\hline 180 & 20 & 24.3 & 10784.1 & 182681 & 15.6 \\
\hline 225 & & & & & \\
\hline 270 & & & & & \\
\hline 315 & & & & & \\
\hline
\end{tabular}

\begin{tabular}{|c|c|c|c|c|c|}
\hline \multicolumn{6}{|l|}{$\begin{array}{l}\text { Second } \\
\text { mode }\end{array}$} \\
\hline $\begin{array}{c}\text { Wind } \\
\text { Direction }\end{array}$ & $\begin{array}{l}\text { Number of } \\
\text { observations } \\
\text { per wind } \\
\text { sector }\end{array}$ & $\begin{array}{c}\text { Location } \\
(\mathrm{nm})\end{array}$ & Amplitude & Area & FWHM \\
\hline \multicolumn{6}{|l|}{0} \\
\hline 45 & 32.3 & 8185.6 & 393600 & 41.9 & 32.3 \\
\hline 90 & 38.9 & 10776.5 & 552790 & 45.4 & 38.9 \\
\hline 135 & 39.5 & 12488.9 & 577503 & 40.2 & 39.5 \\
\hline 180 & 49.3 & 12827.5 & $2 E+06$ & 101.73 & 49.3 \\
\hline \multicolumn{6}{|l|}{225} \\
\hline \multicolumn{6}{|l|}{270} \\
\hline 315 & & & & & \\
\hline
\end{tabular}




\begin{tabular}{|c|c|c|c|c|c|}
\hline \multicolumn{6}{|l|}{ Third mode } \\
\hline $\begin{array}{c}\text { Wind } \\
\text { Direction }\end{array}$ & $\begin{array}{l}\text { Number of } \\
\text { observations } \\
\text { per wind } \\
\text { sector }\end{array}$ & $\begin{array}{c}\text { Location } \\
(\mathrm{nm})\end{array}$ & Amplitude & Area & FWHM \\
\hline \multicolumn{6}{|l|}{0} \\
\hline 45 & 2 & 73.33 & 4964.3 & 795991 & 132.41 \\
\hline 90 & 27 & 87.4 & 4312.4 & 781895 & 151.4 \\
\hline 135 & 16 & 66.26 & 10615.5 & 1467720 & 115.1 \\
\hline 180 & 20 & & & & \\
\hline \multicolumn{6}{|l|}{225} \\
\hline \multicolumn{6}{|l|}{270} \\
\hline 315 & & & & & \\
\hline
\end{tabular}




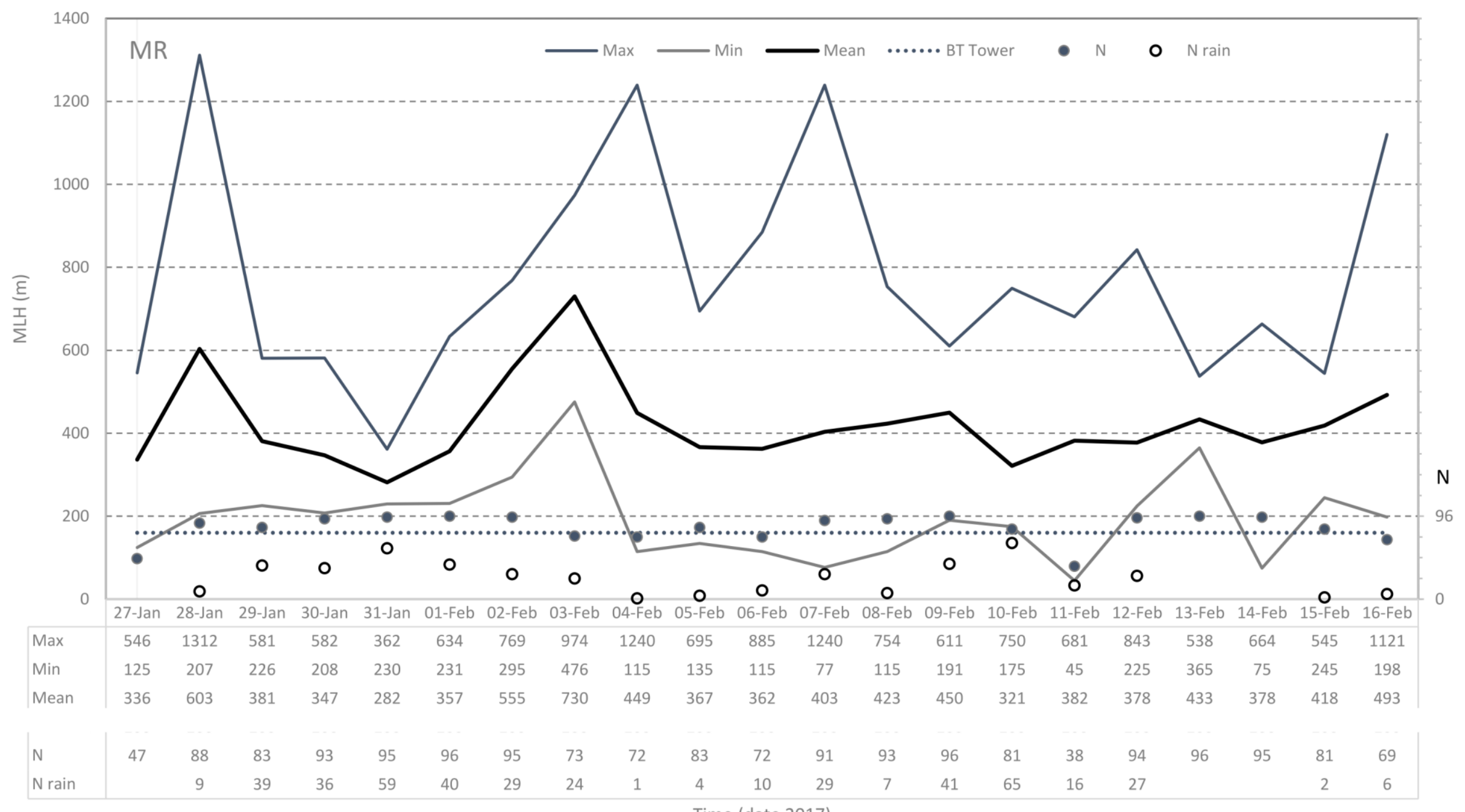

Figure S1: Daily range of mixed layer height (MLH) at Marylebone Road during the campaign period. The number of 15 min periods (N) available for analysis each day and if the number of 15 min periods remaining that still had rain ( $\mathrm{N}$ rain) but the MLH could still be determined. 


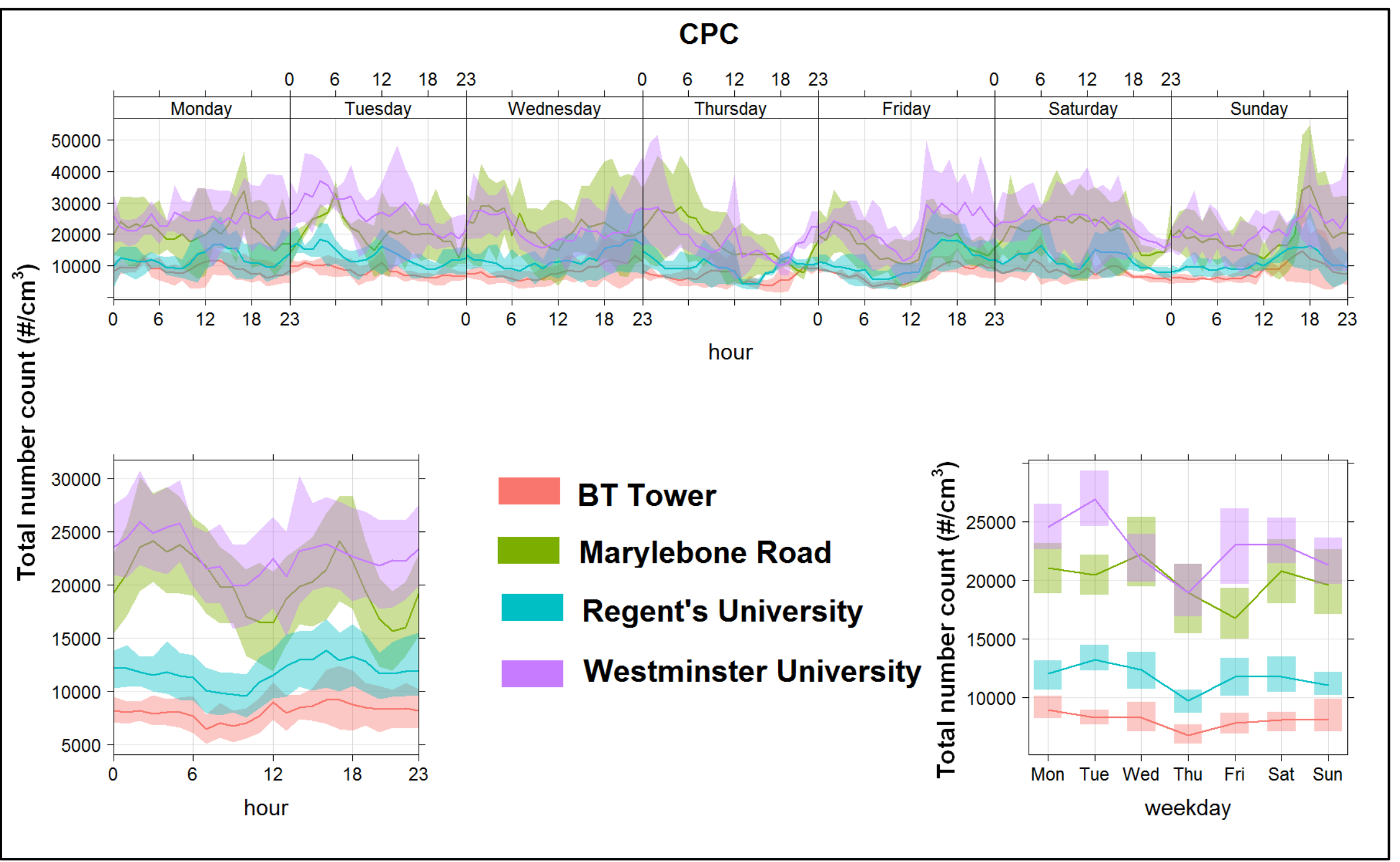

61 Figure S2: Average total number count from CPC instruments by day of week and time of day. 


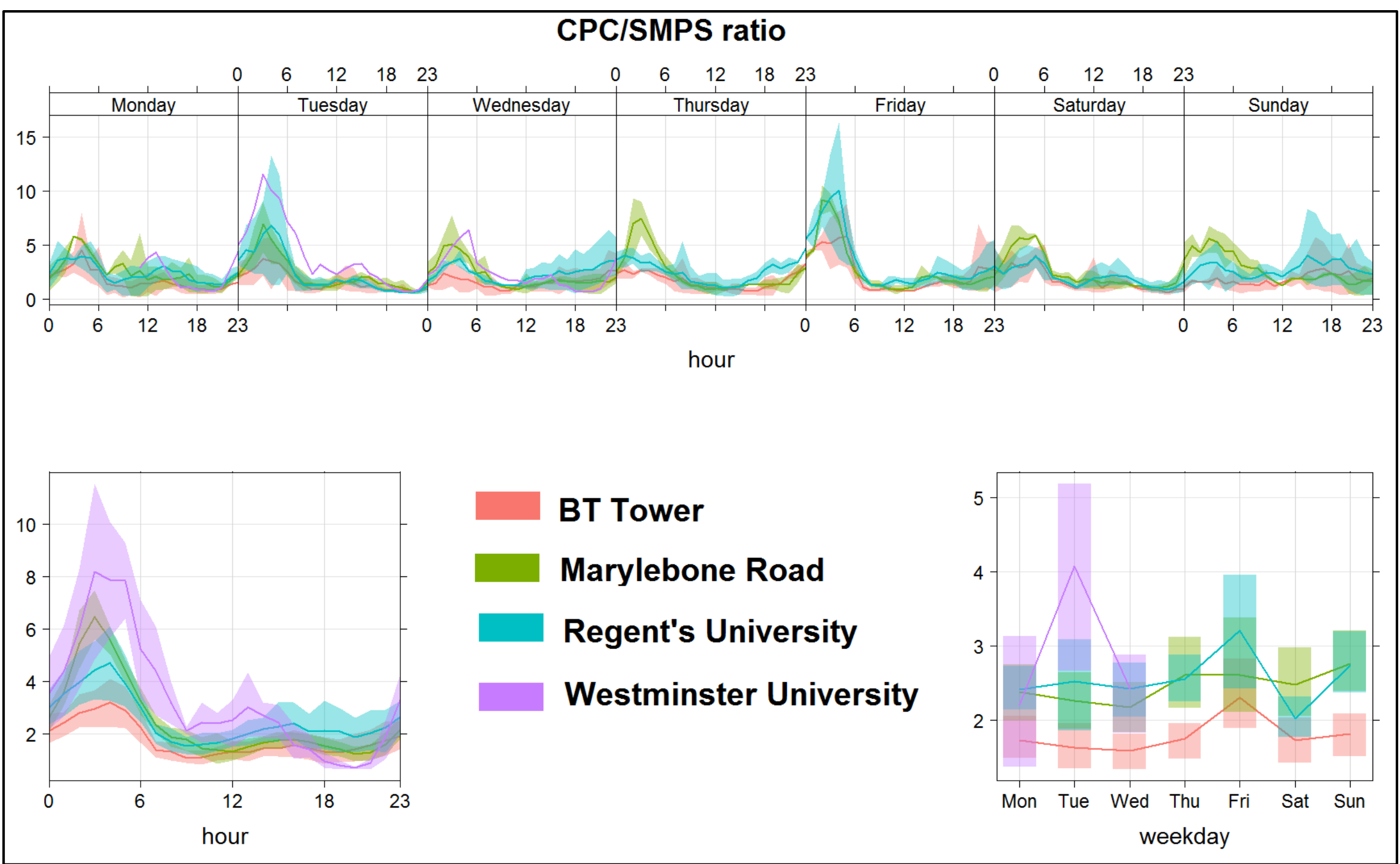

Figure S3: AsFigure S1 but ratio of CPC/SMPS total particle count data. 


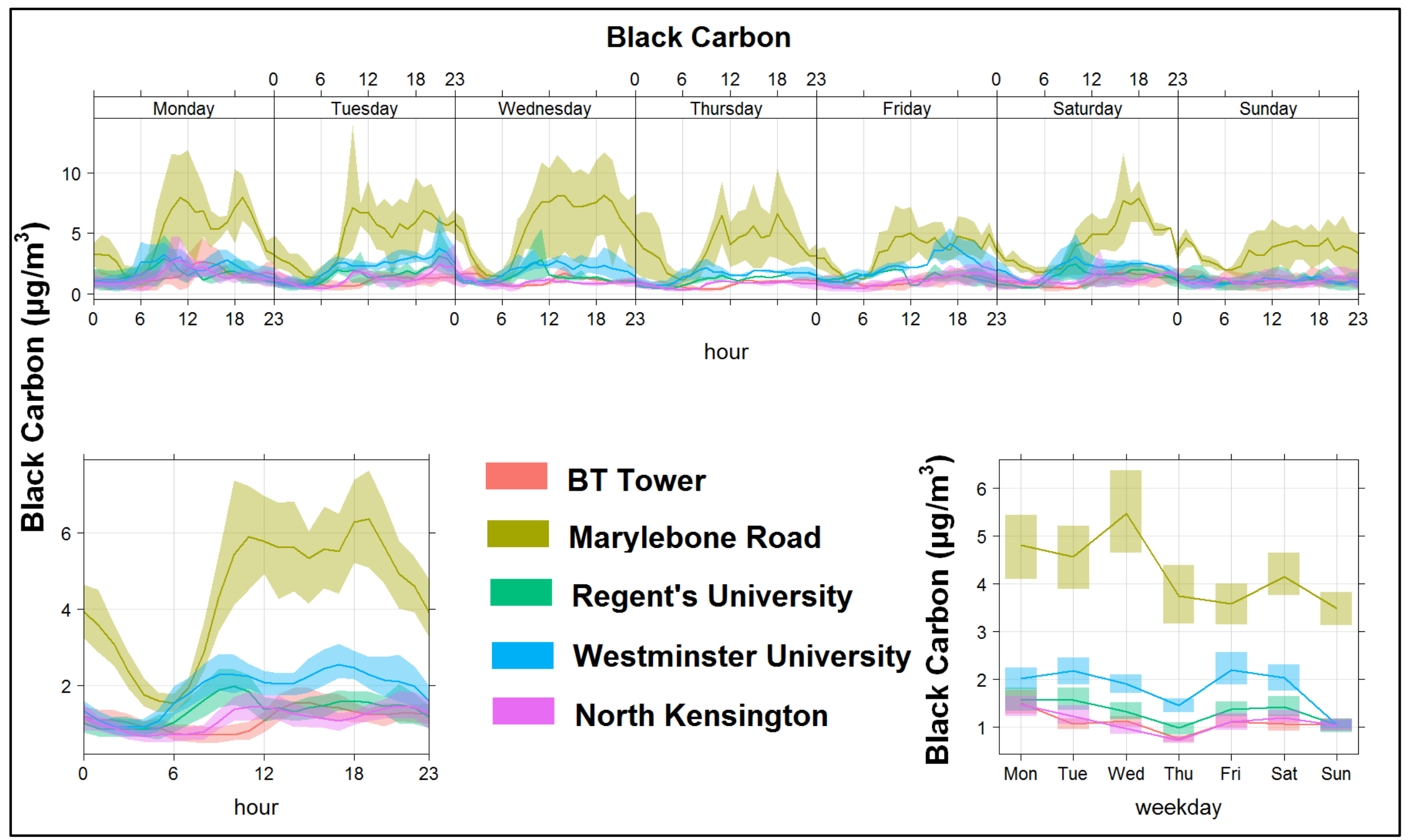

65 Figure S4: As Figure S1, but black carbon concentrations. 


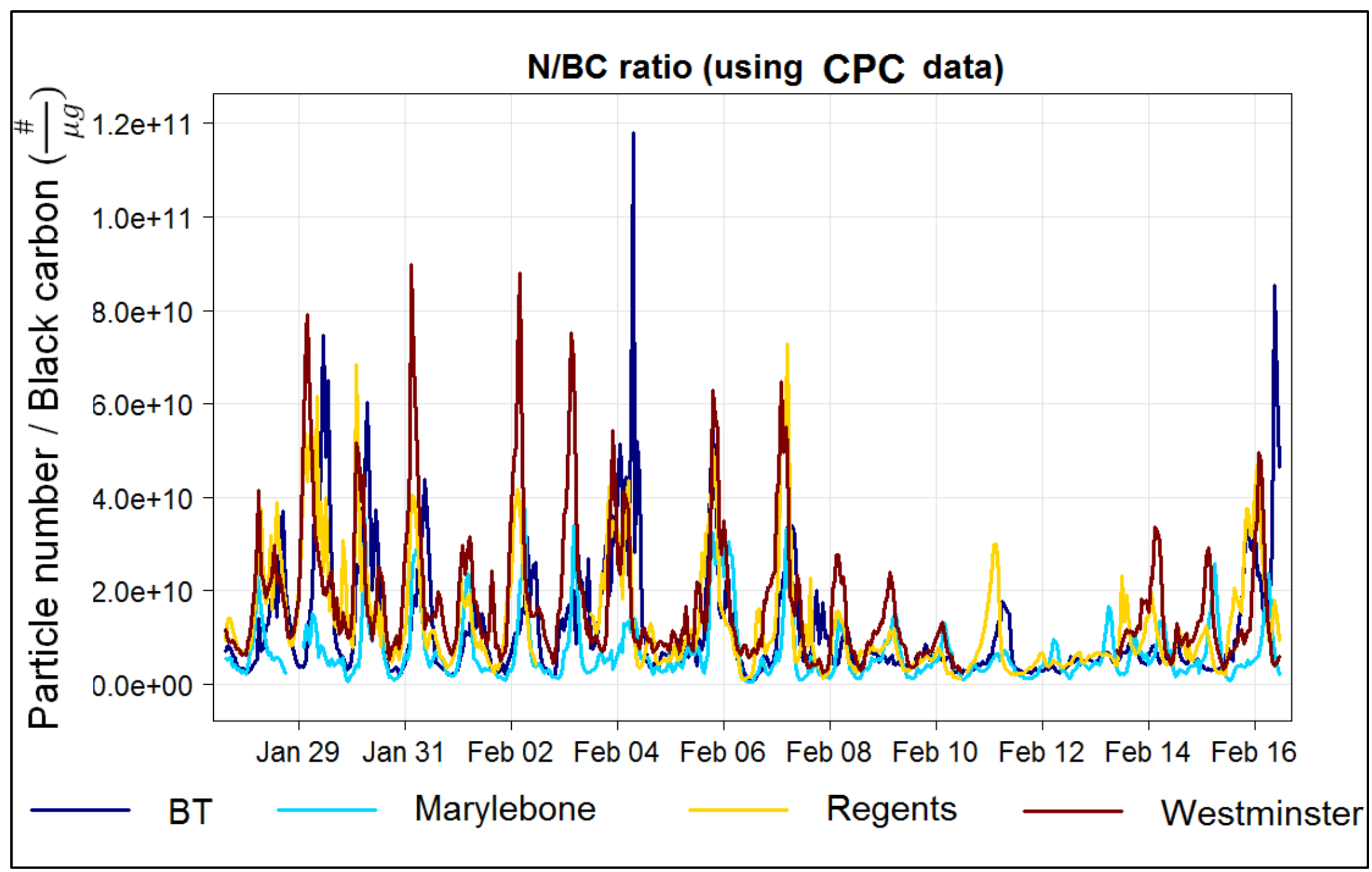

Figure S5: Time series (15 min) of the ratio of total particle number (from CPC) to black carbon for the entire campaign. 


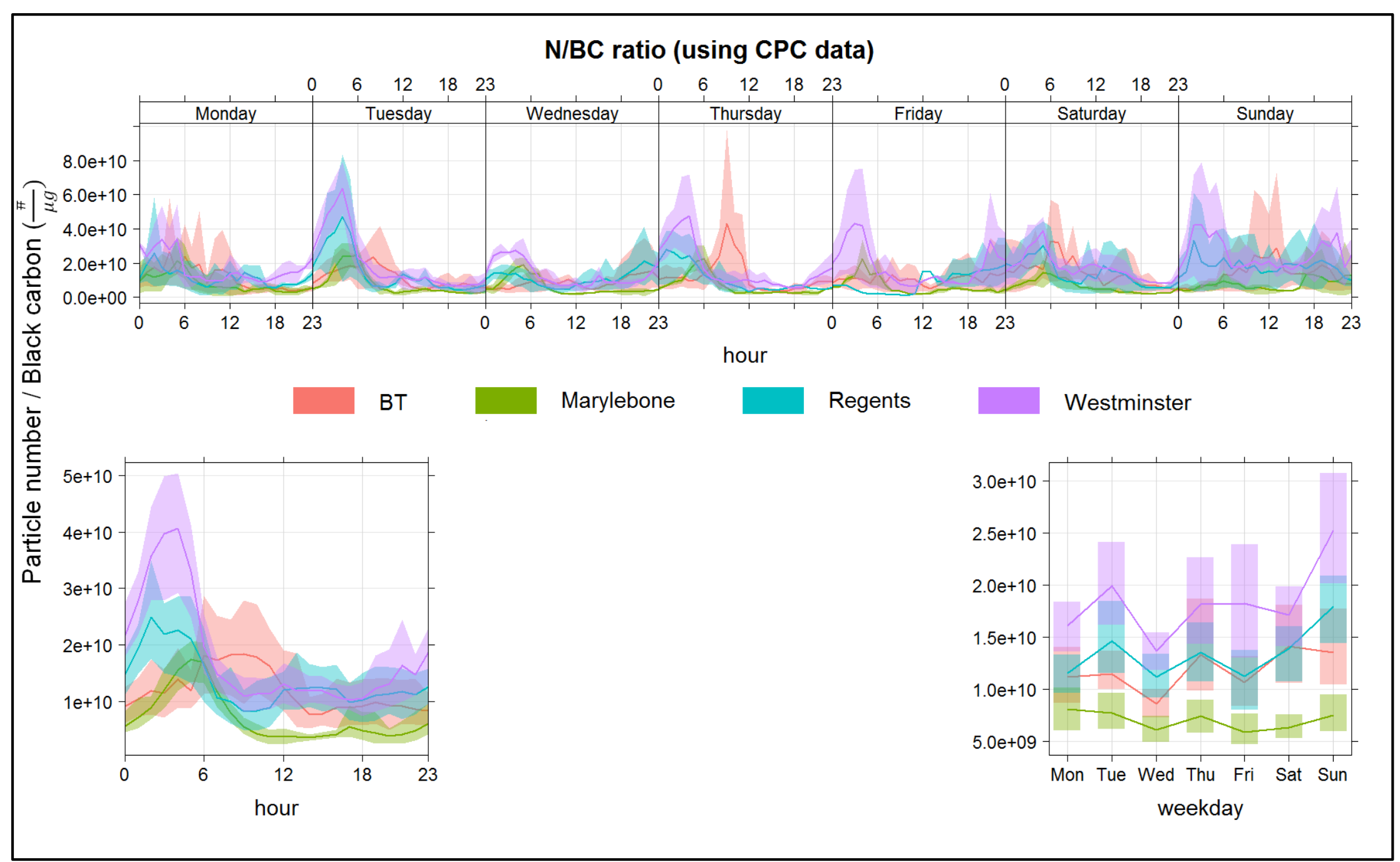

Figure S6: As Figure S1 but ratio of total particle number (from CPC) to black carbon. 


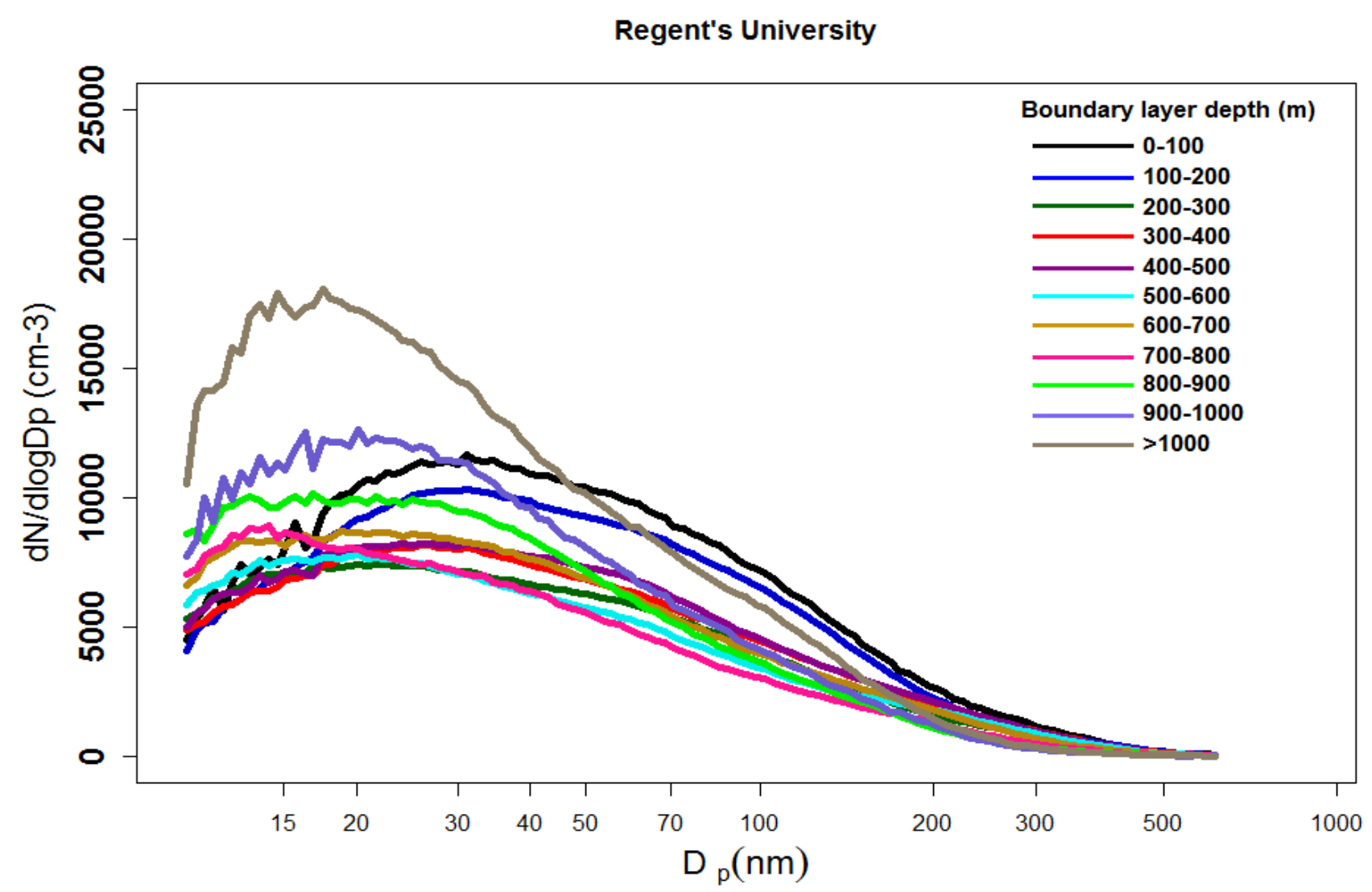

73 Figure S7: Average particle size distributions at Regent's University as a function of mixed layer height (m) as observed at Marylebone Road. Data 74 analysed are $15 \mathrm{~min}$ data. Rain periods are included but account for only $2 \%$ of total time. 


\section{North Kensington}

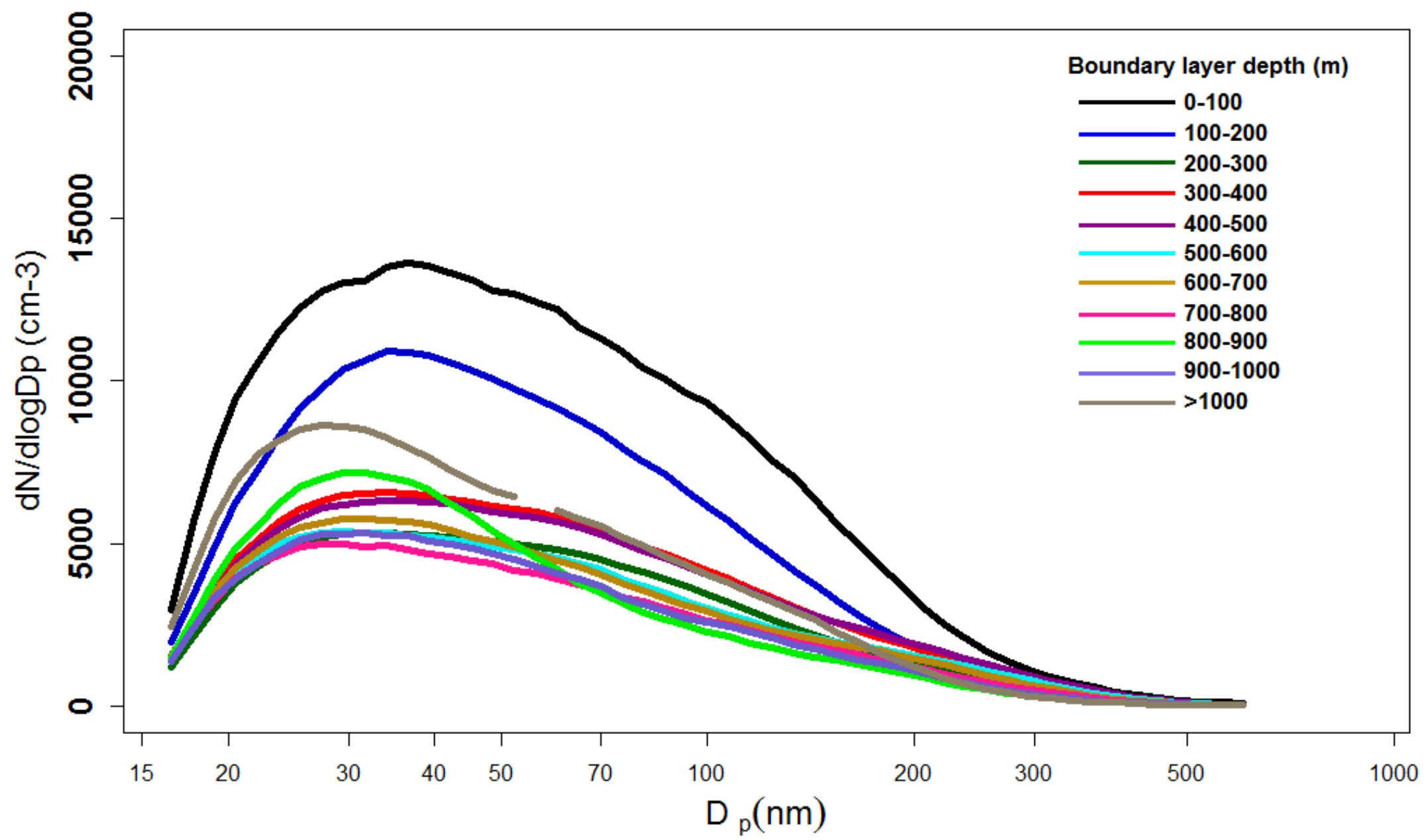




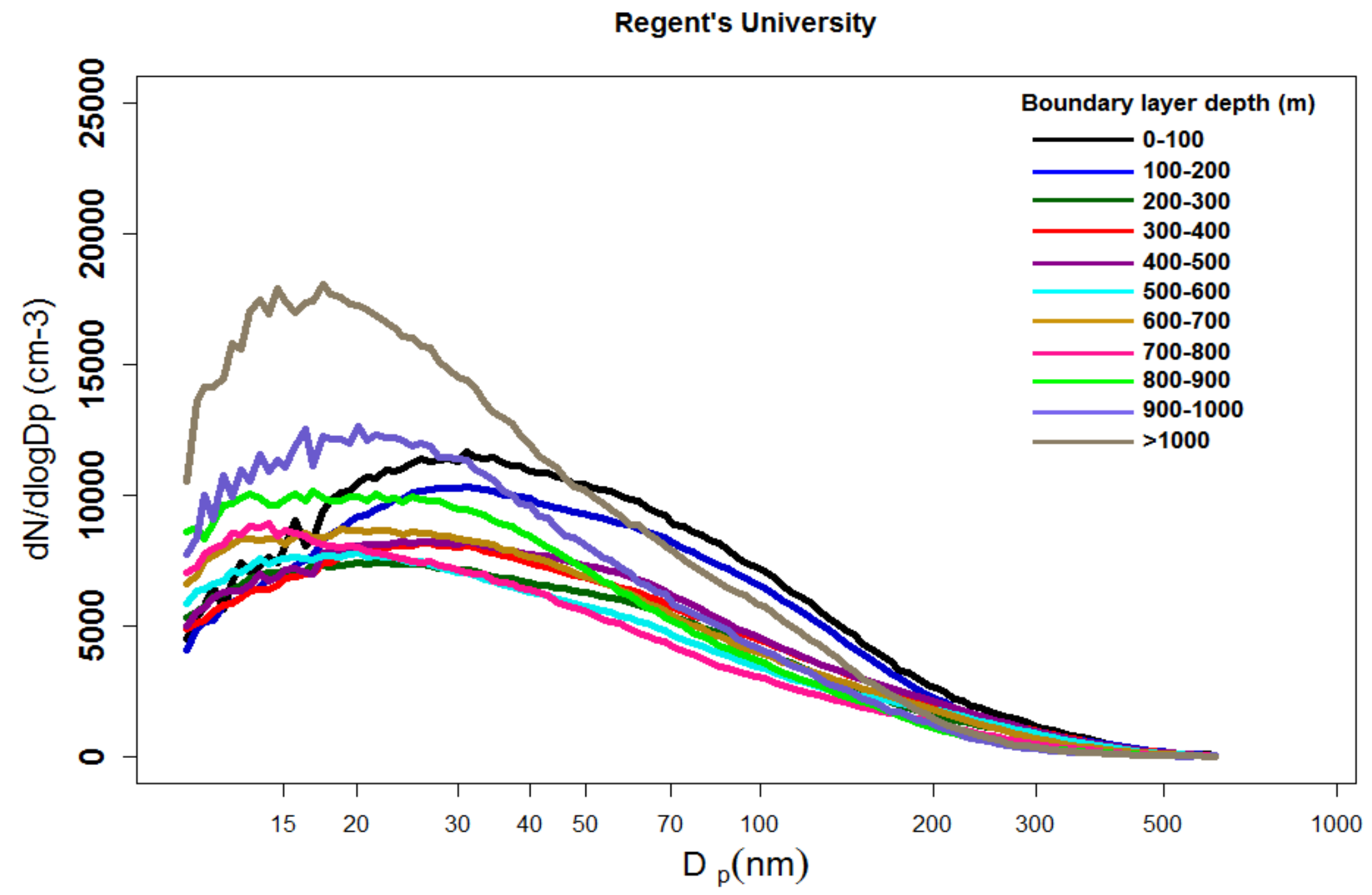

Figure S9: As Figure S6, but for Regent's University. 


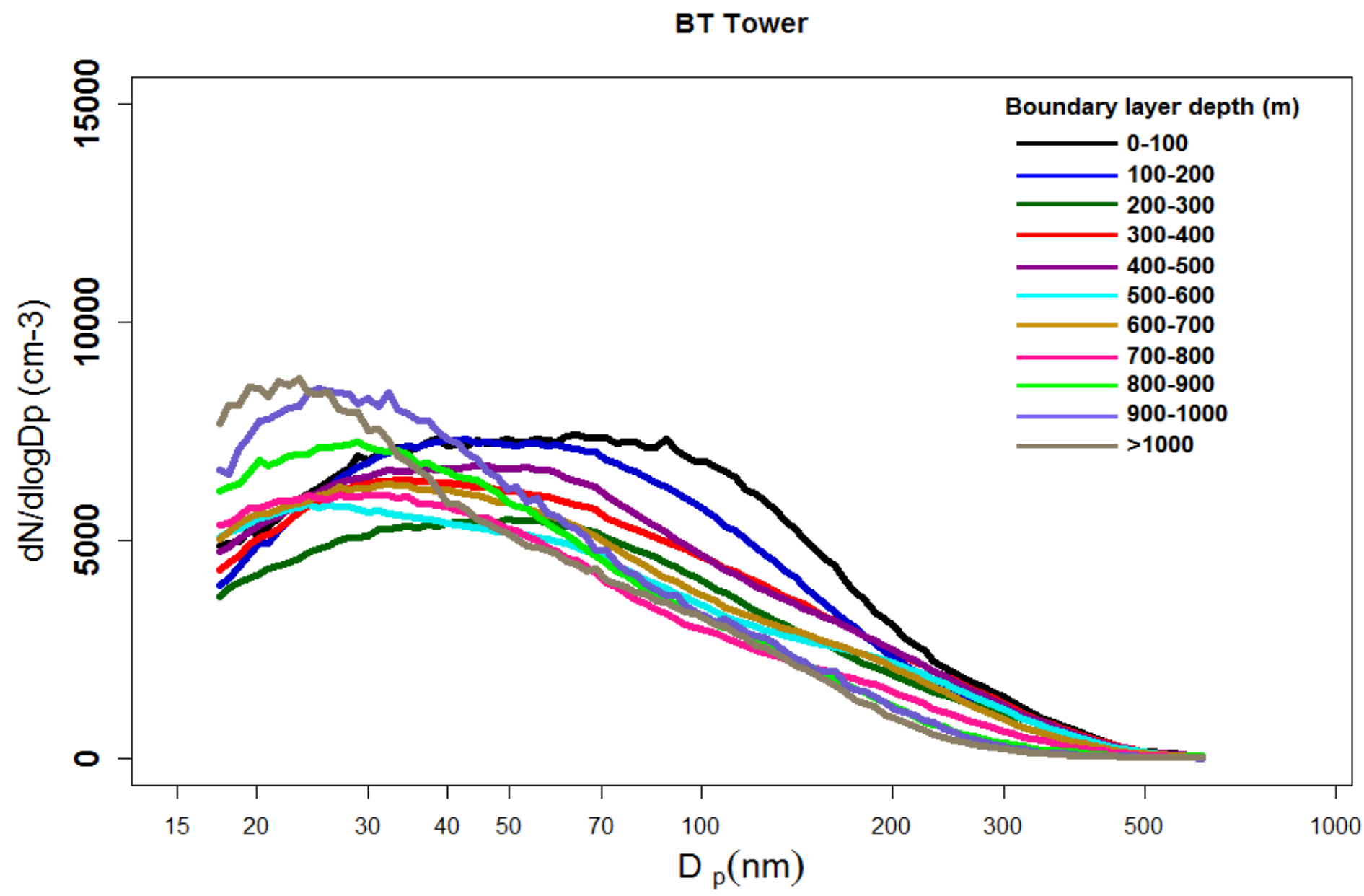

82 Figure S10: As Figure S6, but for BT Tower. 